\title{
ON THE RESURGENCE PROPERTIES OF THE UNIFORM ASYMPTOTIC EXPANSION OF THE INCOMPLETE GAMMA FUNCTION
}

\section{A. B. Olde Daalhuis}

\begin{abstract}
We examine the resurgence properties of the coefficients $c_{r}(\eta)$ appearing in the uniform asymptotic expansion of the incomplete gamma function. For the coefficients $c_{r}(\eta)$, we give an asymptotic approximation as $r \rightarrow \infty$ that is a sum of two incomplete beta functions plus a simple asymptotic series in which the coefficients are again $c_{m}(\eta)$.

The method of this paper is based on the Borel-Laplace transform, which means that next to the asymptotic approximation of $c_{r}(\eta)$, we also obtain an exponentially-improved asymptotic expansion for the incomplete gamma function.
\end{abstract}

\section{Introduction and summary}

Recently it has been shown in many papers that the incomplete gamma function is the basis function for exponential asymptotics. The asymptotic expansion of $\Gamma(a, z)$ as $z \rightarrow \infty$ and $a$ fixed is rather simple. However, in exponential asymptotics, we need the asymptotic properties of $\Gamma(a, z)$ as $a \rightarrow \infty$ and $z=\lambda a, \lambda \neq 0$, a complex constant. In [9] and [11], the uniform asymptotic expansions of the (normalized) incomplete gamma function as $a \rightarrow \infty$ are given as

$$
\begin{aligned}
\frac{\Gamma(a, z)}{\Gamma(a)} & \sim \frac{1}{2} \operatorname{erfc}\left(\eta \sqrt{\frac{1}{2} a}\right)+\frac{e^{-\frac{1}{2} a \eta^{2}}}{\sqrt{2 \pi a}} \sum_{r=0}^{\infty} c_{r}(\eta) a^{-r}, \\
\Gamma(a+1) \frac{e^{-\pi i a}}{2 \pi i} \Gamma\left(-a, z e^{-\pi i}\right) & \sim \frac{1}{2} \operatorname{erfc}\left(-i \eta \sqrt{\frac{1}{2} a}\right)+i \frac{e^{\frac{1}{2} a \eta^{2}}}{\sqrt{2 \pi a}} \sum_{r=0}^{\infty} c_{r}(\eta)(-a)^{-r}
\end{aligned}
$$

where $\eta$ is defined as

$$
\eta=(2(\lambda-1-\ln \lambda))^{\frac{1}{2}}
$$

and

$$
\lambda=\frac{z}{a} .
$$

The complementary error function in (1.1b) will give the Stokes smoothing in exponentially improved asymptotics for integrals and solutions of differential equations. See, for example, $\S 4$ of [8].

Our interest will go to the asymptotics of the coefficients $c_{r}(\eta)$ as $r \rightarrow \infty$. As in most uniform asymptotic expansions, the coefficients are rather complicated functions of their arguments.

Received June 24, 1998, revised September 22, 1998.

1991 Mathematics Subject Classification: Primary 34E05, secondary 33B20.

Key words and phrases: asymptotic expansions, Borel transform, incomplete beta function, incomplete gamma function. 


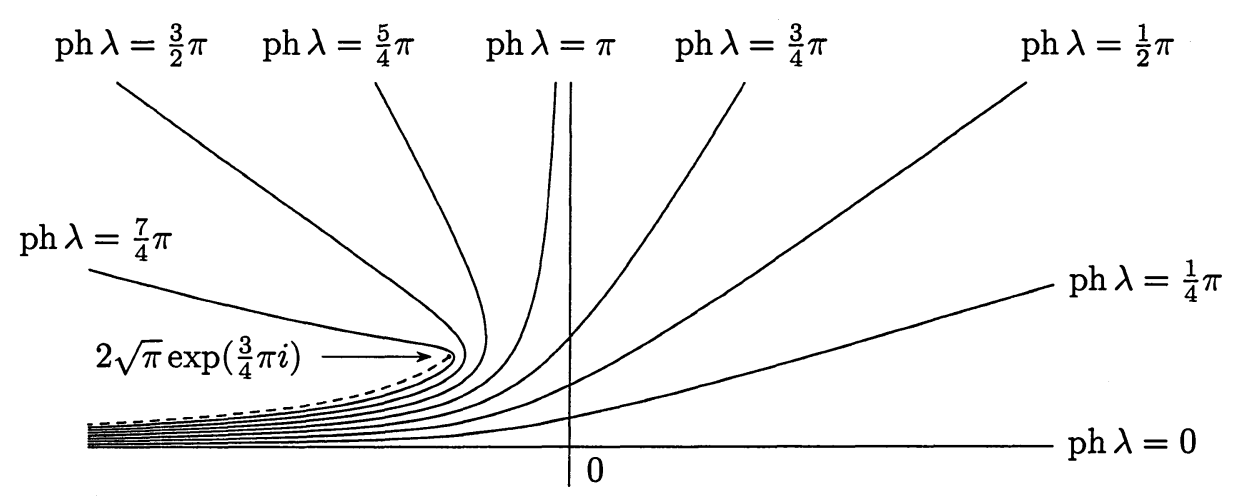

FIGURE 1. $\eta$-plane. The images of rays in the $\lambda$-plane.

First, we want to help the reader with the definition of $\eta$. Since $\lambda-1-\ln \lambda$ has a double root at $\lambda=1$, we prescribe that $\eta(\lambda)$ is analytic in a neighborhood of $\lambda=1$. We take $\eta \sim \lambda-1$ as $\lambda \rightarrow 1$. In Figure 1 , we give the images of the rays $\operatorname{ph} \lambda=\frac{k}{4} \pi, k=0,1, \ldots, 8$. Note that $\lambda=0$ is mapped to $\eta=-\infty$, the half line $0<\lambda<\infty$ is mapped onto the complete real $\eta$-axis, and that the Riemann sheet $0 \leq \operatorname{ph} \lambda<2 \pi$ is mapped onto the upper half plane $\Im \eta \geq 0$ with a cut at the 'half'hyperbola $\{\eta=\alpha+\beta i \mid \alpha \beta=-2 \pi, \alpha \leq-\sqrt{2 \pi}\}$, which is the image of the ray $\operatorname{ph} \lambda=2 \pi$, and which is the dashed line in Figure 1.

Hence, (1.2) is well defined as a mapping from the Riemann sheets $|\operatorname{ph} \lambda|<2 \pi$ to

$$
\mathcal{N}=\{\eta \in \mathbb{C}\} \backslash\{\eta=\alpha+\beta i \mid \alpha \beta= \pm 2 \pi, \quad \alpha \leq-\sqrt{2 \pi}\}
$$

According to [9]-[11], expansions (1.1) hold uniformly in $|z| \in[0, \infty)$, in the domains $|\mathrm{ph} a| \leq \pi-\varepsilon_{1}$ and $|\mathrm{ph} \lambda| \leq 2 \pi-\varepsilon_{2}$ where $\varepsilon_{1}$ and $\varepsilon_{2}$ are small positive numbers. This would mean that the mapping (1.2) is defined on the natural $\lambda$-domain. But, as is noted in [4], the region of validity of (1.1) is smaller. For more details on the region of validity, the reader is referred to the introduction of [4].

The coefficients $c_{r}(\eta)$ are defined by

$$
c_{0}(\eta)=\frac{1}{\lambda-1}-\frac{1}{\eta}, \quad c_{r}(\eta)=\frac{1}{\eta} \frac{d}{d \eta} c_{r-1}(\eta)+\frac{\gamma_{r}}{\lambda-1}, \quad r=1,2, \ldots
$$

where the coefficients $\gamma_{r}$ are those appearing in Stirling's formula

$$
\Gamma(z) \sim \frac{z^{z-\frac{1}{2}} e^{-z}}{\sqrt{2 \pi}} \sum_{r=0}^{\infty}(-)^{r} \gamma_{r} z^{-r}
$$

as $z \rightarrow \infty$.

In [4], a new integral representation is given:

$$
c_{r}(\eta)=\frac{i(-)^{r} \Gamma\left(r+\frac{1}{2}\right)}{(2 \pi)^{\frac{3}{2}}} \oint_{\{1, \lambda\}} \frac{d z}{(z-\lambda)(z-1-\ln z)^{r+\frac{1}{2}}}
$$

where the contour of integration is a loop around 1 and $\lambda$. The authors use this integral representation to obtain an asymptotic expansion of $c_{r}(\eta)$ as $r \rightarrow \infty$. This 
result can be written as

$$
\begin{gathered}
c_{r}(\eta) \sim-\frac{\frac{1}{2} \sqrt{\frac{\pi}{2}}}{\Gamma\left(\frac{1}{2}-r\right)}\left(\frac{1}{2} \eta^{2}+2 \pi i\right)^{-r-\frac{1}{2}} \operatorname{erfc}\left(i \sqrt{r+\frac{1}{2}}\left[\ln \left(\frac{2 \pi i+\frac{1}{2} \eta^{2}}{2 \pi i}\right)\right]^{\frac{1}{2}}\right) \\
-\frac{\frac{1}{2} \sqrt{\frac{\pi}{2}}}{\Gamma\left(\frac{1}{2}-r\right)}\left(\frac{1}{2} \eta^{2}-2 \pi i\right)^{-r-\frac{1}{2}} \operatorname{erfc}\left(-i \sqrt{r+\frac{1}{2}}\left[\ln \left(\frac{2 \pi i-\frac{1}{2} \eta^{2}}{2 \pi i}\right)\right]^{\frac{1}{2}}\right) \\
+\frac{\sqrt{\frac{\pi}{2}}}{\Gamma\left(\frac{1}{2}-r\right)}\left\{(2 \pi i)^{-r-\frac{3}{2}} \sum_{m=0}^{\infty} \frac{\Gamma\left(m+\frac{1}{2}\right) a_{m}^{+}(\lambda)}{\left(r+\frac{1}{2}\right)^{m+\frac{1}{2}}}\right. \\
\left.+(-2 \pi i)^{-r-\frac{3}{2}} \sum_{m=0}^{\infty} \frac{\Gamma\left(m+\frac{1}{2}\right) a_{m}^{-}(\lambda)}{\left(r+\frac{1}{2}\right)^{m+\frac{1}{2}}}\right\}
\end{gathered}
$$

where

$$
\begin{aligned}
a_{m}^{ \pm}(\lambda)=\left(\ln \left\{\frac{2 \pi i \pm \frac{1}{2} \eta^{2}}{2 \pi i}\right\}\right)^{-m-\frac{1}{2}} & -\sum_{j=0}^{m} b_{j}^{(m)}\left( \pm \frac{\eta^{2}}{4 \pi i}\right)^{j-m-\frac{1}{2}} \\
& +e^{\mp \frac{1}{4} \pi i} \sum_{j=0}^{m}(-2 \pi i)^{j+1} \frac{b_{m-j}^{(m)}}{\Gamma\left(j+\frac{1}{2}\right)} c_{j}(\eta)
\end{aligned}
$$

in which the coefficients $b_{j}^{(m)}$ have the generating function

$$
\left(\frac{x}{\ln (1+x)}\right)^{m+\frac{1}{2}}=\sum_{j=0}^{\infty} b_{j}^{(m)} x^{j}
$$

Note that definition (1.9) for the coefficients $a_{m}^{ \pm}$contains the (lower-order) $c_{j}(\eta)$. Hence, there seems to be a resurgence in the asymptotics of $c_{r}(\eta)$ as $r \rightarrow \infty$.

To obtain the resurgence, we will study the Borel transform of the divergent series in (1.1). In $\S 2$, we will define the Borel transform, give a representation that is valid in the whole of $\mathcal{N}$, and obtain the local behavior at the dominant singularities. These singularities are poles and branch points. The location of the singularities will depend on $\eta$, and there will be coalescences of singularities when $\eta=0$, that is, when $\lambda=1$.

In $\S 3$, we will use the dominant singularities of the Borel transform to obtain an asymptotic expansion of $c_{r}(\eta)$ as $r \rightarrow \infty$. Our main result will be:

Theorem. For $\eta \in \mathcal{N}$, we have

$$
\begin{aligned}
c_{r}(\eta) \sim- & \frac{\frac{1}{2} \sqrt{\frac{\pi}{2}}}{\Gamma\left(\frac{1}{2}-r\right)}\left(\frac{1}{2} \eta^{2}+2 \pi i\right)^{-r-\frac{1}{2}} I_{1+\frac{\eta^{2}}{4 \pi i}}\left(r+\frac{1}{2}, \frac{1}{2}\right) \\
& -\frac{\frac{1}{2} \sqrt{\frac{\pi}{2}}}{\Gamma\left(\frac{1}{2}-r\right)}\left(\frac{1}{2} \eta^{2}-2 \pi i\right)^{-r-\frac{1}{2}} I_{1-\frac{\eta^{2}}{4 \pi i}}\left(r+\frac{1}{2}, \frac{1}{2}\right) \\
& +\sum_{n=0}^{\infty} c_{n}(\eta) \Gamma(r-n)\left\{(-2 \pi i)^{n-r-1}+(2 \pi i)^{n-r-1}\right\}
\end{aligned}
$$

as $r \rightarrow \infty$. 
In (1.11), we use the normalized incomplete beta function, which is defined by

$$
I_{x}(p, q)=\frac{1}{B(p, q)} \int_{0}^{x} t^{p-1}(1-t)^{q-1} d t
$$

where $B(p, q)=\Gamma(p) \Gamma(q) / \Gamma(p+q)$ is the beta function. For more details on the incomplete beta function, see $\S 11.3$ in [10].

Hence, we have a simple resurgence relation for the coefficients $c_{r}(\eta)$. Note that due to cancellations in the divergent series in (1.11), the asymptotic expansion of $c_{r}(\eta)$, with $r$ odd, involves only $c_{n}(\eta)$, with $n$ even, and the asymptotic expansion of $c_{r}(\eta)$, with $r$ even, involves only $c_{n}(\eta)$, with $n$ odd. Since the incomplete beta function can be computed via a simple continued fraction ${ }^{1}$, asymptotic result (1.11) is an efficient way to compute the higher-order coefficients $c_{r}(\eta)$. Thus, to use many terms in the asymptotic expansions (1.1), the reader can first compute the lower-order coefficients $c_{r}(\eta)$ and use these results in (1.11) for the computation of the higher-order coefficients, which then can be used in the right-hand sides of (1.1).

In the second half of $\S 3$, we will discuss the asymptotic behavior of the incomplete beta functions that occur in (1.11). From these results, we obtain that in the main part of $\mathcal{N}$, the coefficients $c_{r}(\eta)$ are of the order $\Gamma(r)(2 \pi)^{-r-1}$ as $r \rightarrow \infty$. However, there are two lobes in $\mathcal{N}$ in which one of the terms with an incomplete beta function dominates all the other terms in (1.11), and in these lobes the coefficients $c_{r}(\eta)$ are of the order $\left(\frac{1}{2} \eta^{2} \pm 2 \pi i\right)^{-r-\frac{1}{2}} / \Gamma\left(\frac{1}{2}-r\right)$. This asymptotic behavior also was observed in [4]. At the end of $\S 3$, we give an illustration of this asymptotic behavior.

Finally, in $\S 4$, we give an exponentially improved asymptotic expansion for the incomplete gamma function. The re-expansion is in terms of functions that are more complicated than the incomplete gamma function itself. This is the reason we omit the proof of the results in $\S 4$.

If we compare (1.8) with (1.11), then it is obvious that there are similarities and big differences. The main similarity is that in both equations, there are two loose terms. The loose terms in (1.8) are the error function terms, and they are simpler than the incomplete beta function terms in (1.11). In fact, one could argue that since the incomplete beta function depends on three parameters, it is too complicated to be used as an approximant of $c_{r}(\eta)$. But the incomplete beta functions in (1.11) depend only on two parameters, and one of these parameters is limited to half-integers. Furthermore, the computation of the incomplete beta functions is not very difficult. Again, see [3].

The main difference between (1.8) and (1.11) are the asymptotic series. Note that the asymptotic scales are very different. Furthermore, the coefficients in the asymptotic series are much simpler in (1.11).

Due to the different asymptotic scales, it is not possible to obtain (1.8) directly from (1.11). However, by replacing the incomplete beta functions in (1.11) by asymptotic expansions as $r \rightarrow \infty$ that are uniformly valid in $\eta$, we would obtain the error function terms of (1.8). See (3.15).

\footnotetext{
${ }^{1}$ For a comprehensive discussion of the computation of the incomplete beta function, see [3].
} 


\section{The Borel transform}

Let $\mathcal{P}$ be the complex plane with a cut from 0 to $-\infty$. We take as the Borel transform of the asymptotic series in (1.1)

$$
y(t, \lambda)=\sum_{r=0}^{\infty} c_{r}(\eta) \Gamma\left(\frac{1}{2}-r\right) t^{r-\frac{1}{2}}
$$

This series is only convergent for $t$ in a neighborhood of 0 . To obtain a representation in a larger $t$-domain, we use (1.7) in (2.1) and obtain

$$
y(t, \lambda)=t^{-\frac{1}{2}} \frac{i}{2 \sqrt{2 \pi}} \oint_{\{1, \lambda\}} \frac{(z-1-\ln z)^{\frac{1}{2}}}{(z-\lambda)(z-1-\ln z-t)} d z .
$$

In the derivation of this integral representation, we need the restriction $\mid t /(z-1-$ $\ln z) \mid<1$ for all $z$ on the contour of integration. This is not a problem for $t$ in a neighborhood of 0 . This integral representation is, of course, valid in a much larger $t$ domain. Hence, we can replace the restriction $|t /(z-1-\ln z)|<1$ by $t \neq z-1-\ln z$ for all $z$ on the contour of integration.

We deform this contour of integration to a path that begins at $z=-\infty$, encircles $z=0$ once in a negative sense, and returns to its starting point. In this way, we obtain the integral representation

$$
y(t, \lambda)=t^{-\frac{1}{2}} \frac{i}{2 \sqrt{2 \pi}} \int_{-\infty}^{(0-)} \frac{(z-1-\ln z)^{\frac{1}{2}}}{(z-\lambda)(z-1-\ln z-t)} d z
$$

which is valid for $\operatorname{ph} \lambda \in(-\pi, \pi)$ and $t \in \mathcal{P}, \Im t \in(-\pi, \pi)$. In the following lemma, we give a representation that is valid in a larger $\lambda$-domain.

Lemma 1. For $\operatorname{ph} \lambda \in(-2 \pi, 2 \pi)$ and $t \in \mathcal{P}$, we have

$$
\begin{aligned}
y(t, \lambda) & =\frac{1}{2} \sqrt{\frac{\pi}{2}} t^{-\frac{1}{2}}\left(\frac{(\lambda-1+2 \pi i-\ln \lambda)^{\frac{1}{2}}}{t-(\lambda-1-\ln \lambda+2 \pi i)}+\frac{(\lambda-1-2 \pi i-\ln \lambda)^{\frac{1}{2}}}{t-(\lambda-1-\ln \lambda-2 \pi i)}\right) \\
- & \frac{1}{2} \sqrt{\frac{\pi}{2}}(\lambda-1-\ln \lambda)^{\frac{1}{2}}\left(\frac{(t-2 \pi i)^{-\frac{1}{2}}}{t-(\lambda-1-\ln \lambda+2 \pi i)}+\frac{(t+2 \pi i)^{-\frac{1}{2}}}{t-(\lambda-1-\ln \lambda-2 \pi i)}\right) \\
+ & h_{+}(t, \lambda)-h_{-}(t, \lambda)+s_{+}(t, \lambda)-s_{-}(t, \lambda)
\end{aligned}
$$

where

$$
\begin{aligned}
h_{ \pm}(t, \lambda)= & \frac{i}{2 \sqrt{2 \pi}(t-(\lambda-1-\ln \lambda \pm 2 \pi i))}\left\{\left(\frac{\lambda-1 \pm 2 \pi i-\ln \lambda}{t}\right)^{\frac{1}{2}}\right. \\
& \left.\quad \times \ln \left(\frac{1-\left(\frac{\lambda-1-\ln \lambda}{\lambda-1 \pm 2 \pi i-\ln \lambda}\right)^{\frac{1}{2}}}{1+\left(\frac{\lambda-1-\ln \lambda}{\lambda-1 \pm 2 \pi i-\ln \lambda}\right)^{\frac{1}{2}}}\right)-\left(\frac{\lambda-1-\ln \lambda}{t \mp 2 \pi i}\right)^{\frac{1}{2}} \ln \left(\frac{1-\left(\frac{t \mp 2 \pi i}{t}\right)^{\frac{1}{2}}}{1+\left(\frac{t \mp 2 \pi i}{t}\right)^{\frac{1}{2}}}\right)\right\}
\end{aligned}
$$

and

$$
s_{ \pm}(t, \lambda)=\frac{t^{-\frac{1}{2}}}{4 \pi \sqrt{2 \pi}} \int_{0}^{\infty} \int_{\mathcal{C}(\lambda)} \frac{(u \pm 2 \pi i)^{\frac{1}{2}} u^{-\frac{1}{2}}(z-1-\ln z)^{\frac{1}{2}}}{(u-(t \mp 2 \pi i))(z-\lambda)(z-1-\ln z-u)} d z d u
$$

where the $z$-contour $\mathcal{C}(\lambda)$ is defined in Figure $2 b, c$. 


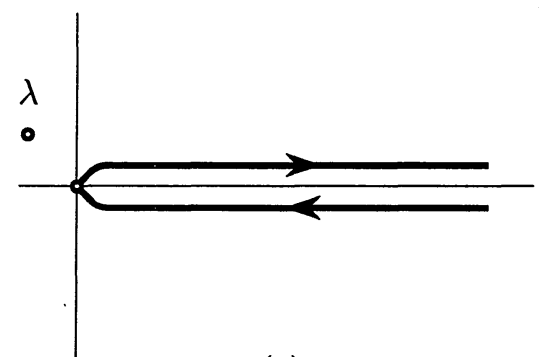

(a)

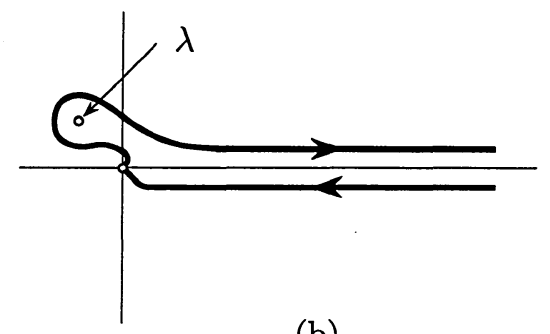

(b)



(c)

Figure 2. (a) Contour $\mathcal{C}$; (b) Contour $\mathcal{C}(\lambda)$ when $\operatorname{ph} \lambda \in(0,2 \pi)$; (c) Contour $\mathcal{C}(\lambda)$ when ph $\lambda \in(-2 \pi, 0)$.

Proof. We start with the restrictions

$\Re t>0, \quad \Im t \in(-2 \pi, 2 \pi), \quad \operatorname{ph} \lambda \in(0, \pi), \quad$ and $\operatorname{ph}(\lambda-1-\ln \lambda) \in\left(0, \frac{1}{2} \pi\right)$.

The branch point at $z=0$ in the integral in (2.2) is integrable. We split the contour of integration in (2.2) into a contour from $\infty e^{\pi i}$ to the origin plus a contour from the origin to $\infty e^{-\pi i}$. We rotate the first contour to the contour from $\infty e^{2 \pi i}$ to the origin and the second contour to the contour from the origin to $\infty e^{-2 \pi i}$. During the second rotation, we encounter a pole at $z=\lambda e^{-2 \pi i}$. We note that when $\operatorname{ph} \lambda \in(-\pi, \pi)$, then $\left(\lambda e^{ \pm 2 \pi i}-1-\ln \left(\lambda e^{ \pm 2 \pi i}\right)\right)^{1 / 2}=-(\lambda-1 \mp 2 \pi i-\ln \lambda)^{1 / 2}$ where on the right-hand side we use the usual square root. In this way, we obtain the integral representation

$$
\begin{aligned}
y(t, \lambda)= & \sqrt{\frac{\pi}{2}}\left(\frac{\lambda-1+2 \pi i-\ln \lambda}{t}\right)^{\frac{1}{2}} \frac{1}{t-(\lambda-1-\ln \lambda+2 \pi i)} \\
& +t^{-\frac{1}{2}} \frac{1}{2 i \sqrt{2 \pi}} \int_{0}^{\infty} \frac{(z-1+2 \pi i-\ln z)^{\frac{1}{2}}}{(z-\lambda)(z-1+2 \pi i-\ln z-t)} d z \\
& -t^{-\frac{1}{2}} \frac{1}{2 i \sqrt{2 \pi}} \int_{0}^{\infty} \frac{(z-1-2 \pi i-\ln z)^{\frac{1}{2}}}{(z-\lambda)(z-1-2 \pi i-\ln z-t)} d z
\end{aligned}
$$

which is valid for ph $\lambda \in(0,2 \pi)$. To obtain a double integral representation for the first integral on the right-hand side of (2.7), we use the substitution $u=z-1-\ln z$ and write $\frac{d z}{d u} /(z-\lambda)$ as a contour integral. For more details, see [2]. We obtain

$$
\begin{aligned}
t^{-\frac{1}{2}} \frac{1}{2 i \sqrt{2 \pi}} \int_{0}^{\infty} \frac{(z-1+2 \pi i-\ln z)^{\frac{1}{2}}}{(z-\lambda)(z-1+2 \pi i-\ln z-t)} d z \\
\quad=\frac{t^{-\frac{1}{2}}}{4 \pi \sqrt{2 \pi}} \int_{0}^{\infty} \int_{\mathcal{C}} \frac{(u+2 \pi i)^{\frac{1}{2}} u^{-\frac{1}{2}}(z-1-\ln z)^{\frac{1}{2}}}{(u-(t-2 \pi i))(z-\lambda)(z-1-\ln z-u)} d z d u
\end{aligned}
$$


where $\mathcal{C}$ is defined in Figure 2a. We deform contour $\mathcal{C}$ to contour $\mathcal{C}(\lambda)$ and obtain

$$
\begin{aligned}
t^{-\frac{1}{2}} \frac{1}{2 i \sqrt{2 \pi}} \int_{0}^{\infty} \frac{(z-1+2 \pi i-\ln z)^{\frac{1}{2}}}{(z-\lambda)(z-1+2 \pi i-\ln z-t)} d z=s_{+}(t, \lambda) \\
\quad+(\lambda-1-\ln \lambda)^{\frac{1}{2}} \frac{t^{-\frac{1}{2}}}{2 i \sqrt{2 \pi}} \int_{0}^{\infty} \frac{(u+2 \pi i)^{\frac{1}{2}} u^{-\frac{1}{2}}}{(u-(t-2 \pi i))(u-(\lambda-1-\ln \lambda))} d u
\end{aligned}
$$

The integral on the right-hand side of (2.9) can be expressed in terms of elementary functions. We use the transformation $u=2 \pi i v$ and rotate the path of integration from the negative imaginary axis onto the positive real axis:

$$
\begin{gathered}
(\lambda-1-\ln \lambda)^{\frac{1}{2}} \frac{t^{-\frac{1}{2}}}{2 i \sqrt{2 \pi}} \int_{0}^{\infty} \frac{(u+2 \pi i)^{\frac{1}{2}} u^{-\frac{1}{2}}}{(u-(t-2 \pi i))(u-(\lambda-1-\ln \lambda))} d u \\
=-\frac{\sqrt{\frac{\pi}{2}}\left(\frac{\lambda-1+2 \pi i-\ln \lambda}{t}\right)^{\frac{1}{2}}}{t-(\lambda-1-\ln \lambda+2 \pi i)}-\frac{(\lambda-1-\ln \lambda)^{\frac{1}{2}} t^{-\frac{1}{2}}}{4 \pi \sqrt{2 \pi}} \\
\quad \times \int_{0}^{\infty} \frac{(v+1)^{\frac{1}{2}} v^{-\frac{1}{2}}}{\left(v-\frac{t-2 \pi i}{2 \pi i}\right)\left(v-\frac{\lambda-1-\ln \lambda}{2 \pi i}\right)} d v .
\end{gathered}
$$

The first term on the right-hand side originates from the pole at $v=(\lambda-1-\ln \lambda) /(2 \pi i)$ which is due to restrictions (2.6) in the fourth quadrant. The substitution $v=z^{2} / 1-z^{2}$ leads to

$$
\begin{aligned}
& -\frac{(\lambda-1-\ln \lambda)^{\frac{1}{2}} t^{-\frac{1}{2}}}{4 \pi \sqrt{2 \pi}} \int_{0}^{\infty} \frac{(v+1)^{\frac{1}{2}} v^{-\frac{1}{2}}}{\left(v-\frac{t-2 \pi i}{2 \pi i}\right)\left(v-\frac{\lambda-1-\ln \lambda}{2 \pi i}\right)} d v \\
& =\frac{(\lambda-1-\ln \lambda)^{\frac{1}{2}} t^{-\frac{1}{2}} i}{\sqrt{2 \pi}(t-(\lambda-1-\ln \lambda+2 \pi i))}\left[\int_{0}^{1} \frac{d z}{z^{2}-\frac{\lambda-1-\ln \lambda}{\lambda-1+2 \pi i-\ln \lambda}}-\int_{0}^{1} \frac{d z}{z^{2}-\frac{t-2 \pi i}{t}}\right] \\
& \quad=\frac{i}{2 \sqrt{2 \pi}(t-(\lambda-1-\ln \lambda+2 \pi i))}\left\{\left(\frac{\lambda-1+2 \pi i-\ln \lambda}{t}\right)^{\frac{1}{2}}\right. \\
& \left.\times\left[\ln \frac{1-\left(\frac{\lambda-1-\ln \lambda}{\lambda-1+2 \pi i-\ln \lambda}\right)^{1 / 2}}{1+\left(\frac{\lambda-1-\ln \lambda}{\lambda-1+2 \pi i-\ln \lambda}\right)^{1 / 2}}-\pi i\right]-\left(\frac{\lambda-1-\ln \lambda}{t-2 \pi i}\right)^{\frac{1}{2}}\left[\ln \frac{1-\left(\frac{t-2 \pi i}{t}\right)^{1 / 2}}{1+\left(\frac{t-2 \pi i}{t}\right)^{1 / 2}}-\pi i\right]\right\}
\end{aligned}
$$

where we used (2.6) to obtain the correct contributions from the end-point $z=0$. Combining (2.9)-(2.11) leads to

$$
\begin{aligned}
& t^{-\frac{1}{2}} \frac{1}{2 i \sqrt{2 \pi}} \int_{0}^{\infty} \frac{(z-1+2 \pi i-\ln z)^{\frac{1}{2}}}{(z-\lambda)(z-1+2 \pi i-\ln z-t)} d z \\
& =-\frac{\frac{1}{2} \sqrt{\frac{\pi}{2}}\left(\frac{\lambda-1+2 \pi i-\ln \lambda}{t}\right)^{\frac{1}{2}}}{t-(\lambda-1+2 \pi i-\ln \lambda)}-\frac{\frac{1}{2} \sqrt{\frac{\pi}{2}}\left(\frac{\lambda-1-\ln \lambda}{t-2 \pi i}\right)^{\frac{1}{2}}}{t-(\lambda-1+2 \pi i-\ln \lambda)} \\
& \quad+h_{+}(t, \lambda)+s_{+}(t, \lambda) .
\end{aligned}
$$

In a similar manner, we can show that

$$
t^{-\frac{1}{2}} \frac{1}{2 i \sqrt{2 \pi}} \int_{0}^{\infty} \frac{(z-1-2 \pi i-\ln z)^{\frac{1}{2}}}{(z-\lambda)(z-1-2 \pi i-\ln z-t)} d z
$$




$$
\begin{aligned}
=- & \frac{\frac{1}{2} \sqrt{\frac{\pi}{2}}\left(\frac{\lambda-1-2 \pi i-\ln \lambda}{t}\right)^{\frac{1}{2}}}{t-(\lambda-1-2 \pi i-\ln \lambda)}+\frac{\frac{1}{2} \sqrt{\frac{\pi}{2}}\left(\frac{\lambda-1-\ln \lambda}{t+2 \pi i}\right)^{\frac{1}{2}}}{t-(\lambda-1-2 \pi i-\ln \lambda)} \\
& +h_{-}(t, \lambda)+s_{-}(t, \lambda) .
\end{aligned}
$$

Hence, (2.3) follows from (2.7), (2.12), and (2.13). Analytic continuation shows us that (2.3) is valid for $\operatorname{ph} \lambda \in(-2 \pi, 2 \pi)$ and $t \in \mathcal{P}$.

The main property that we need from the Borel transform $y(t, \lambda)$ is the local behavior at the singularities that are nearest to the branch point $t=0$. The first two terms on the right-hand side of (2.3) are rather simple singularities. Notice that in the second term, the branch point and the pole coalesce when $\lambda=1$. Although the definition (2.4) of $h_{ \pm}(t, \lambda)$ is rather complicated, these functions have only one singularity in $\mathcal{P}$ and that singularity is a branch point at $t=0$.

The remaining problem in this section is to find the singularities of $s_{ \pm}(t, \lambda)$. We define for $\alpha>0$ the functions

$$
s_{ \pm}(t, \lambda, \alpha)=\frac{t^{-\frac{1}{2}}}{4 \pi \sqrt{2 \pi}} \int_{0}^{\alpha} \int_{\mathcal{C}(\lambda)} \frac{(u \pm 2 \pi i)^{\frac{1}{2}} u^{-\frac{1}{2}}(z-1-\ln z)^{\frac{1}{2}}}{(u-(t \mp 2 \pi i))(z-\lambda)(z-1-\ln z-u)} d z d u .
$$

We note that the functions $s_{ \pm}(t, \lambda)-s_{ \pm}(t, \lambda, \alpha)$ are analytic for $|t \mp 2 \pi i|<\alpha$. Hence, by taking $\alpha$ large enough, the singular behavior at the singularities that are the nearest to $t=0$ will be the same for $s_{ \pm}(t, \lambda)$ and $s_{ \pm}(t, \lambda, \alpha)$. These singularities originate from the end-point $u=0$. We expand the factor $1 /(z-1-\ln z-u)$ in a Taylor series at $u=0$ and obtain

$$
\begin{aligned}
s_{ \pm}(t, \lambda, \alpha) & =\frac{t^{-\frac{1}{2}}}{4 \pi \sqrt{2 \pi}} \sum_{n=0}^{\infty} \int_{0}^{\alpha} \frac{(u \pm 2 \pi i)^{\frac{1}{2}} u^{n-\frac{1}{2}}}{u-(t \mp 2 \pi i)} d u \int_{\mathcal{C}(\lambda)} \frac{d z}{(z-\lambda)(z-1-\ln z)^{n+\frac{1}{2}}} \\
& =-\sum_{n=0}^{\infty} c_{n}(\eta) \Gamma\left(\frac{1}{2}-n\right) \frac{t^{-\frac{1}{2}}}{2 \pi i} \int_{0}^{\alpha} \frac{(u \pm 2 \pi i)^{\frac{1}{2}} u^{n-\frac{1}{2}}}{u-(t \mp 2 \pi i)} d u
\end{aligned}
$$

where we used (1.7) to compute the $z$-integral.

In the next lemma, $r e g(t-2 \pi i)$ denotes a function that is regular (or analytic) in a neighborhood of $t=2 \pi i$.

\section{Lemma 2.}

$$
t^{-\frac{1}{2}} \int_{0}^{\alpha} \frac{(u \pm 2 \pi i)^{\frac{1}{2}} u^{n-\frac{1}{2}}}{u-(t \mp 2 \pi i)} d u=\pi(-)^{n}( \pm 2 \pi i-t)^{n-\frac{1}{2}}+\operatorname{reg}(t \mp 2 \pi i) .
$$

Proof.

$$
\begin{aligned}
t^{-\frac{1}{2}} & \int_{0}^{\alpha} \frac{(u \pm 2 \pi i)^{\frac{1}{2}} u^{n-\frac{1}{2}}}{u-(t \mp 2 \pi i)} d u=\int_{0}^{\alpha} \frac{\left(\frac{u-(t \mp 2 \pi i)}{t}+1\right)^{\frac{1}{2}} u^{n-\frac{1}{2}}}{u-(t \mp 2 \pi i)} d u \\
& =\int_{0}^{\alpha} \frac{u^{n-\frac{1}{2}} d u}{u-(t \mp 2 \pi i)}+\frac{1}{2 t} \int_{0}^{\alpha} \int_{0}^{1}\left(\{u-(t \mp 2 \pi i)\} \frac{w}{t}+1\right)^{-\frac{1}{2}} u^{n-\frac{1}{2}} d w d u \\
& =\int_{0}^{\alpha} \frac{u^{n-\frac{1}{2}} d u}{u-(t \mp 2 \pi i)}+\operatorname{reg}(t \mp 2 \pi i)
\end{aligned}
$$


We use (15.3.1) and (15.3.7) of [1] and obtain

$$
\begin{aligned}
\int_{0}^{\alpha} \frac{u^{n-\frac{1}{2}} d u}{u-(t \mp 2 \pi i)} & =\frac{\alpha^{n+\frac{1}{2}}}{ \pm 2 \pi i-t} \int_{0}^{1} \frac{v^{n-\frac{1}{2}} d v}{1-v \frac{\alpha}{t \mp 2 \pi i}} \\
& =\frac{\alpha^{n+\frac{1}{2}}}{( \pm 2 \pi i-t)\left(n+\frac{1}{2}\right)}{ }_{2} F_{1}\left(1, n+\frac{1}{2} ; n+\frac{3}{2} ; \frac{\alpha}{t \mp 2 \pi i}\right) \\
& =\pi(-)^{n}( \pm 2 \pi i-t)^{n-\frac{1}{2}}+\frac{\alpha^{n-\frac{1}{2}}}{n-\frac{1}{2}}{ }_{2} F_{1}\left(1, \frac{1}{2}-n ; \frac{3}{2}-n ; \frac{t \mp 2 \pi i}{\alpha}\right) \\
& =\pi(-)^{n}( \pm 2 \pi i-t)^{n-\frac{1}{2}}+\operatorname{reg}(t \mp 2 \pi i) .
\end{aligned}
$$

Hence,

$$
s_{ \pm}(t, \lambda)=\frac{i}{2} \sum_{n=0}^{\infty} c_{n}(\eta) \Gamma\left(\frac{1}{2}-n\right)(-)^{n}( \pm 2 \pi i-t)^{n-\frac{1}{2}}+r e g(t \mp 2 \pi i) .
$$

\section{Late coefficients asymptotics}

To obtain an asymptotic expansion for $c_{r}(\eta)$ as $r \rightarrow \infty$, we use Darboux's method. Hence, we use (2.1) and obtain

$$
c_{r}(\eta)=\frac{1}{2 \pi i \Gamma\left(\frac{1}{2}-r\right)} \int_{-\infty}^{\left(0^{+}\right)} y(t, \lambda) t^{-r-\frac{1}{2}} d t .
$$

The main contribution in the asymptotics of $c_{r}(\eta)$ as $r \rightarrow \infty$ comes from the singularity of $y(t, \lambda)$ that is nearest to $t=0$. In the previous section, we showed that the nearest singularities are at $t_{\lambda \pm}=\lambda-1 \pm 2 \pi i-\ln \lambda$ and $t_{1 \pm}= \pm 2 \pi i$. Hence, the dominant asymptotic behavior in the bounded regions $|\lambda-1 \pm 2 \pi i-\ln \lambda|<2 \pi$ when $t_{\lambda \pm}$ is nearest will be very different from the dominant asymptotic behaviour in the region $|\lambda-1 \pm 2 \pi i-\ln \lambda|>2 \pi$ when $t_{1 \pm}$ is nearest. To obtain an asymptotic expansion that is uniformly valid in $\operatorname{ph} \lambda \in(-2 \pi, 2 \pi)$, we use (2.3) in (3.1) and obtain

$$
\begin{aligned}
c_{r}(\eta)= & -\frac{\frac{1}{2} \sqrt{\frac{\pi}{2}}}{\Gamma\left(\frac{1}{2}-r\right)}\left((\lambda-1+2 \pi i-\ln \lambda)^{-r-\frac{1}{2}}+(\lambda-1-2 \pi i-\ln \lambda)^{-r-\frac{1}{2}}\right) \\
& -\frac{\frac{1}{2} \sqrt{\frac{\pi}{2}}(\lambda-1-\ln \lambda)^{\frac{1}{2}}}{2 \pi i \Gamma\left(\frac{1}{2}-r\right)}\left(\int_{-\infty}^{(0+)} \frac{(t-2 \pi i)^{-\frac{1}{2}} t^{-r-\frac{1}{2}}}{t-(\lambda-1+2 \pi i-\ln \lambda)} d t\right. \\
& \left.\quad+\int_{-\infty}^{(0+)} \frac{(t+2 \pi i)^{-\frac{1}{2}} t^{-r-\frac{1}{2}}}{t-(\lambda-1-2 \pi i-\ln \lambda)} d t\right) \\
& +\frac{1}{2 \pi i \Gamma\left(\frac{1}{2}-r\right)} \int_{-\infty}^{(0+)}\left(h_{+}(t, \lambda)-h_{-}(t, \lambda)\right) t^{-r-\frac{1}{2}} d t \\
& +\frac{1}{2 \pi i \Gamma\left(\frac{1}{2}-r\right)} \int_{-\infty}^{(0+)} s_{+}(t, \lambda) t^{-r-\frac{1}{2}} d t-\frac{1}{2 \pi i \Gamma\left(\frac{1}{2}-r\right)} \int_{-\infty}^{(0+)} s_{-}(t, \lambda) t^{-r-\frac{1}{2}} d t .
\end{aligned}
$$


The first two integrals are contour integral representations for the incomplete beta function. From (11.42) in [10], we obtain

$$
\begin{aligned}
& \frac{(\lambda-1-\ln \lambda)^{\frac{1}{2}}}{2 \pi i}\left(\int_{-\infty}^{(0+)} \frac{(t-2 \pi i)^{-\frac{1}{2}} t^{-r-\frac{1}{2}}}{t-(\lambda-1+2 \pi i-\ln \lambda)} d t\right. \\
& \left.\quad+\int_{-\infty}^{(0+)} \frac{(t+2 \pi i)^{-\frac{1}{2}} t^{-r-\frac{1}{2}}}{t-(\lambda-1-2 \pi i-\ln \lambda)} d t\right)= \\
& =-(\lambda-1+2 \pi i-\ln \lambda)^{-r-\frac{1}{2}} I_{\frac{\lambda-1-\ln \lambda}{-2 \pi i}\left(\frac{1}{2}, r+\frac{1}{2}\right)} \\
& \quad-(\lambda-1-2 \pi i-\ln \lambda)^{-r-\frac{1}{2}} I_{\frac{\lambda-1-\ln \lambda}{2 \pi i}}\left(\frac{1}{2}, r+\frac{1}{2}\right) .
\end{aligned}
$$

Since the only singularity of $h_{ \pm}(t, \lambda)$ in the bounded $t$-plane is a branch point at $t=0$, the third integral in (3.3) can be estimated by

$$
\frac{1}{2 \pi i \Gamma\left(\frac{1}{2}-r\right)} \int_{-\infty}^{(0+)}\left(h_{+}(t, \lambda)-h_{-}(t, \lambda)\right) t^{-r-\frac{1}{2}} d t=\frac{1}{\Gamma\left(\frac{1}{2}-r\right)} \mathcal{O}\left(A^{-r}\right) \quad \text { as } r \rightarrow \infty
$$

where $A$ is an arbitrary fixed positive constant.

For the fourth integral in (3.2), we use (2.19) and obtain

$$
\begin{aligned}
& \frac{1}{2 \pi i \Gamma\left(\frac{1}{2}-r\right)} \int_{-\infty}^{(0+)} s_{+}(t, \lambda) t^{-r-\frac{1}{2}} d t=\frac{1}{2 \pi i \Gamma\left(\frac{1}{2}-r\right)} \int_{i \infty}^{\left(2 \pi i^{-}\right)} s_{+}(t, \lambda) t^{-r-\frac{1}{2}} d t \\
& \sim \frac{1}{4 \pi} \sum_{n=0}^{\infty}(-)^{n} c_{n}(\eta) \frac{\Gamma\left(\frac{1}{2}-n\right)}{\Gamma\left(\frac{1}{2}-r\right)} \int_{i \infty}^{\left(2 \pi i^{-}\right)}(2 \pi i-t)^{n-\frac{1}{2}} t^{-r-\frac{1}{2}} d t
\end{aligned}
$$

Note that

$$
\begin{aligned}
\int_{i \infty}^{\left(2 \pi i^{-}\right)}(2 \pi i-t)^{n-\frac{1}{2}} t^{-r-\frac{1}{2}} d t & =(2 \pi)^{n-r} e^{\frac{1}{2} \pi i(n+r+1)} \int_{\infty}^{\left(0^{+}\right)}(\tau+1)^{n-\frac{1}{2}} \tau^{-r-\frac{1}{2}} d \tau \\
& =2(-)^{n} i(-2 \pi i)^{n-r} B\left(\frac{1}{2}-r, r-n\right)
\end{aligned}
$$

Hence,

$$
\frac{1}{2 \pi i \Gamma\left(\frac{1}{2}-r\right)} \int_{-\infty}^{(0+)} s_{+}(t, \lambda) t^{-r-\frac{1}{2}} d t \sim \sum_{n=0}^{\infty} \frac{c_{n}(\eta)}{(-2 \pi i)^{r-n+1}} \Gamma(r-n) \quad \text { as } r \rightarrow \infty .
$$

Similarly,

$$
\frac{1}{2 \pi i \Gamma\left(\frac{1}{2}-r\right)} \int_{-\infty}^{(0+)} s_{-}(t, \lambda) t^{-r-\frac{1}{2}} d t \sim-\sum_{n=0}^{\infty} \frac{c_{n}(\eta)}{(2 \pi i)^{r-n+1}} \Gamma(r-n) \quad \text { as } r \rightarrow \infty .
$$

Since we can absorb the left-hand side of (3.5) into the right-hand side of (3.8) and (3.9), we have shown that

$$
\begin{aligned}
& c_{r}(\eta) \sim-\frac{\frac{1}{2} \sqrt{\frac{\pi}{2}}}{\Gamma\left(\frac{1}{2}-r\right)}\left((\lambda-1+2 \pi i-\ln \lambda)^{-r-\frac{1}{2}}+(\lambda-1-2 \pi i-\ln \lambda)^{-r-\frac{1}{2}}\right) \\
& +\frac{\frac{1}{2} \sqrt{\frac{\pi}{2}}}{\Gamma\left(\frac{1}{2}-r\right)}\left\{(\lambda-1+2 \pi i-\ln \lambda)^{-r-\frac{1}{2}} \frac{I_{\lambda-1-\ln \lambda}}{-2 \pi i}\left(\frac{1}{2}, r+\frac{1}{2}\right)\right. \\
& \left.+(\lambda-1-2 \pi i-\ln \lambda)^{-r-\frac{1}{2}} I_{\frac{\lambda-1-\ln \lambda}{2 \pi i}}\left(\frac{1}{2}, r+\frac{1}{2}\right)\right\}
\end{aligned}
$$




$$
+\sum_{n=0}^{\infty} c_{n}(\eta) \Gamma(r-n)\left\{(-2 \pi i)^{n-r-1}+(2 \pi i)^{n-r-1}\right\}
$$

as $r \rightarrow \infty$. This expansion is valid for ph $\lambda \in(-2 \pi, 2 \pi)$.

When we apply the identity $I_{1-x}(q, p)=1-I_{x}(p, q)$, we can present the final result as

$$
\begin{aligned}
c_{r}(\eta) \sim- & \frac{\frac{1}{2} \sqrt{\frac{\pi}{2}}}{\Gamma\left(\frac{1}{2}-r\right)}\left(\frac{1}{2} \eta^{2}+2 \pi i\right)^{-r-\frac{1}{2}} I_{1+\frac{\eta^{2}}{4 \pi i}}\left(r+\frac{1}{2}, \frac{1}{2}\right) \\
& -\frac{\frac{1}{2} \sqrt{\frac{\pi}{2}}}{\Gamma\left(\frac{1}{2}-r\right)}\left(\frac{1}{2} \eta^{2}-2 \pi i\right)^{-r-\frac{1}{2}} I_{1-\frac{\eta^{2}}{4 \pi i}}\left(r+\frac{1}{2}, \frac{1}{2}\right) \\
& +\sum_{n=0}^{\infty} c_{n}(\eta) \Gamma(r-n)\left\{(-2 \pi i)^{n-r-1}+(2 \pi i)^{n-r-1}\right\}
\end{aligned}
$$

as $r \rightarrow \infty$.

To obtain a better understanding of which terms of the right-hand side of (3.11) are dominant in which sectors of the complex $\eta$-plane, we give the asymptotic behavior of the first term.

In the case $-\pi \leq \operatorname{ph} \eta \leq \frac{1}{2} \pi$ and $\eta$ bounded away from the origin, we have

$$
\begin{aligned}
& -\frac{\frac{1}{2} \sqrt{\frac{\pi}{2}}}{\Gamma\left(\frac{1}{2}-r\right)}\left(\frac{1}{2} \eta^{2}+2 \pi i\right)^{-r-\frac{1}{2}} I_{1+\frac{\eta^{2}}{4 \pi i}}\left(r+\frac{1}{2}, \frac{1}{2}\right) \\
& \quad=-\frac{\frac{1}{2} \sqrt{\frac{\pi}{2}}}{\Gamma\left(\frac{1}{2}-r\right)} \frac{\left(\frac{1}{2} \eta^{2}+2 \pi i\right)^{-r-\frac{1}{2}}}{B\left(\frac{1}{2}, r+\frac{1}{2}\right)} \int_{0}^{1+\frac{\eta^{2}}{4 \pi i}} t^{r-\frac{1}{2}}(1-t)^{-\frac{1}{2}} d t \\
& \quad \sim-\frac{\frac{1}{2} \sqrt{\frac{\pi}{2}}}{\Gamma\left(\frac{1}{2}-r\right)} \frac{\left(\frac{1}{2} \eta^{2}+2 \pi i\right)^{-r-\frac{1}{2}}}{B\left(\frac{1}{2}, r+\frac{1}{2}\right)} \int_{0}^{1+\frac{\eta^{2}}{4 \pi i}} t^{r-\frac{1}{2}}\left(-\frac{\eta^{2}}{4 \pi i}\right)^{-\frac{1}{2}} d t \\
& \quad \sim \frac{\Gamma(r)}{\eta}(-2 \pi i)^{-r-1}
\end{aligned}
$$

as $r \rightarrow \infty$.

In the case $\frac{1}{2} \pi \leq \operatorname{ph} \eta \leq \pi$ and $\eta$ bounded away from the origin, we use that $I_{x^{2}}\left(\frac{1}{2}, r+\frac{1}{2}\right)$ is an odd function of $x$ and obtain

$$
\begin{aligned}
I_{1+\frac{\eta^{2}}{4 \pi i}}\left(r+\frac{1}{2}, \frac{1}{2}\right) & =1-I_{-\frac{\eta^{2}}{4 \pi i}\left(\frac{1}{2}, r+\frac{1}{2}\right)} \\
& =1+I_{-} \frac{\left(\eta e^{-\pi i}\right)^{2}}{4 \pi i}\left(\frac{1}{2}, r+\frac{1}{2}\right) \\
& =2-I_{1+\frac{\left(\eta e^{-\pi i}\right)^{2}}{4 \pi i}}\left(r+\frac{1}{2}, \frac{1}{2}\right) .
\end{aligned}
$$

Now we can use (3.12) and obtain

$$
\begin{aligned}
-\frac{\frac{1}{2} \sqrt{\frac{\pi}{2}}}{\Gamma\left(\frac{1}{2}-r\right)}\left(\frac{1}{2} \eta^{2}+2 \pi i\right)^{-r-\frac{1}{2}} & I_{1+\frac{\eta^{2}}{4 \pi i}}\left(r+\frac{1}{2}, \frac{1}{2}\right) \\
& \sim-\frac{\sqrt{\frac{\pi}{2}}}{\Gamma\left(\frac{1}{2}-r\right)}\left(\frac{1}{2} \eta^{2}+2 \pi i\right)^{-r-\frac{1}{2}}+\frac{\Gamma(r)}{\eta}(-2 \pi i)^{-r-1}
\end{aligned}
$$

as $r \rightarrow \infty$. 
Finally, in the case of $\eta$ in the neighborhood of the origin we write

$$
\begin{aligned}
& -\frac{\frac{1}{2} \sqrt{\frac{\pi}{2}}}{\Gamma\left(\frac{1}{2}-r\right)}\left(\frac{1}{2} \eta^{2}+2 \pi i\right)^{-r-\frac{1}{2}} I_{1+\frac{\eta^{2}}{4 \pi i}}\left(r+\frac{1}{2}, \frac{1}{2}\right) \\
& \quad=-\frac{\frac{1}{2} \sqrt{\frac{\pi}{2}}}{\Gamma\left(\frac{1}{2}-r\right)} \frac{\left(\frac{1}{2} \eta^{2}+2 \pi i\right)^{-r-\frac{1}{2}}}{B\left(\frac{1}{2}, r+\frac{1}{2}\right)} \int_{-\frac{\eta^{2}}{4 \pi i}}^{1}(1-t)^{r-\frac{1}{2}} t^{-\frac{1}{2}} d t \\
& \quad=-\frac{\frac{1}{2} \sqrt{\frac{\pi}{2}}}{\Gamma\left(\frac{1}{2}-r\right)} \frac{\left(\frac{1}{2} \eta^{2}+2 \pi i\right)^{-r-\frac{1}{2}}}{B\left(\frac{1}{2}, r+\frac{1}{2}\right)} \int_{\ln \left(\frac{2 \pi i}{\eta^{2} / 2+2 \pi i}\right)}^{\infty} e^{-\left(r+\frac{1}{2}\right) s} s^{-\frac{1}{2}}\left(\frac{s}{1-e^{-s}}\right)^{\frac{1}{2}} d s \\
& \quad \sim-\frac{\frac{1}{2} \sqrt{\frac{\pi}{2}}}{\Gamma\left(\frac{1}{2}-r\right)} \frac{\left(\frac{1}{2} \eta^{2}+2 \pi i\right)^{-r-\frac{1}{2}}}{B\left(\frac{1}{2}, r+\frac{1}{2}\right)} \int_{\ln \left(\frac{2 \pi i}{\eta^{2} / 2+2 \pi i}\right)}^{\infty} e^{-\left(r+\frac{1}{2}\right) s} s^{-\frac{1}{2}} d s \\
& \quad \sim-\frac{\frac{1}{2} \sqrt{\frac{\pi}{2}}}{\Gamma\left(\frac{1}{2}-r\right)}\left(\frac{1}{2} \eta^{2}+2 \pi i\right)^{-r-\frac{1}{2}} \operatorname{erfc}\left(\left(r+\frac{1}{2}\right)^{1 / 2}\left(\ln \frac{2 \pi i}{\frac{1}{2} \eta^{2}+2 \pi i}\right)^{\frac{1}{2}}\right)
\end{aligned}
$$

as $r \rightarrow \infty$ where $\operatorname{erfc}(x)$ is the complementary error function. ${ }^{2}$

From (3.12), (3.14), (3.15), and the asymptotics of $\operatorname{erfc}(x)$ as $x \rightarrow \infty$, we obtain that the first term of the right-hand side of (3.11) is of the same order as the first term of the divergent series of (3.11), except in the region of the sector $\frac{1}{2} \pi \leq \operatorname{ph} \eta \leq \pi$ where the first term of the right-hand side of (3.14) dominates the second term. This region is given by

$$
\left\{\eta=-\alpha+\beta i \mid \alpha, \beta>0 \text { and }\left(\alpha^{2}+\beta^{2}\right)^{2}<16 \alpha \beta \pi\right\} .
$$

Similarly, the second term of the right-hand side of (3.11) is of the same order as the first term of the divergent series, except in the region

$$
\left\{\eta=-\alpha-\beta i \mid \alpha, \beta>0 \text { and }\left(\alpha^{2}+\beta^{2}\right)^{2}<16 \alpha \beta \pi\right\}
$$

where we have

$$
-\frac{\frac{1}{2} \sqrt{\frac{\pi}{2}}}{\Gamma\left(\frac{1}{2}-r\right)}\left(\frac{1}{2} \eta^{2}-2 \pi i\right)^{-r-\frac{1}{2}} I_{1-\frac{\eta^{2}}{4 \pi i}}\left(r+\frac{1}{2}, \frac{1}{2}\right) \sim-\frac{\sqrt{\frac{\pi}{2}}}{\Gamma\left(\frac{1}{2}-r\right)}\left(\frac{1}{2} \eta^{2}-2 \pi i\right)^{-r-\frac{1}{2}}
$$

as $r \rightarrow \infty$.

As an illustration of the change of dominant asymptotic behavior of $c_{r}(\eta)$ as a function of $\eta$, we give Figure 3. In the example, we take $r=11$ and $\eta=3 \exp (\theta i)$. We note that in the sector $0<\theta<\frac{1}{2} \pi$, the modulus of $c_{r}(\eta)$ is almost constant, and in the sector $\frac{1}{2} \pi<\theta<\pi$, it varies with $\theta$ and has a maximum at $\theta=\frac{3}{4} \pi$.

To give a "numerical proof" of (1.11), we use 6 terms in the sum of the right-hand side of (1.11) (as we noted before, only 3 of the 6 terms are non-zero), and we evaluate the incomplete beta functions via the continued fractions. The absolute and relative errors are shown in Figure 3.

\section{Exponentially improved asymptotics of $\Gamma(a, z)$}

In $\S 2$, we obtained the local behavior of the Borel transform $y(t, \lambda)$ at the dominant singularities. In the previous section, we used this information to obtain an asymptotic

\footnotetext{
${ }^{2}$ To obtain a full uniform asymptotic expansion of the left-hand side of (3.15), the reader can use the first three lines of (3.15) and exercise 6 of chapter 7 of [12].
} 




FiguRE 3. An example with $r=11$ and $\eta=3 \exp (\theta i)$.

expansion for the late coefficients in the divergent series in (1.1). As in [6], we also can use this information to obtain a re-expansion for the remainder term $R_{N}(a, \eta)$ in

$$
\sqrt{2 \pi a} e^{\frac{1}{2} a \eta^{2}}\left(\frac{\Gamma(a, z)}{\Gamma(a)}-\frac{1}{2} \operatorname{erfc}\left(\eta \sqrt{\frac{1}{2} a}\right)\right)=\sum_{r=0}^{N-1} c_{r}(\eta) a^{-r}+R_{N}(a, \eta)
$$

The method will be very similar to the method used in [6]. Thus, we would use the Laplace transform of $y(t, \lambda)$ as an integral representation of the left-hand side of (4.1), substitute a truncated version of (2.1) into this Laplace transform to obtain (4.1), and use Taylor's theorem to obtain a double integral representation for $R_{N}(a, \eta)$. The deformation of the contours in this double integral will give us

$$
\begin{aligned}
& R_{N}(a, \lambda)=\frac{1}{(2 \pi i)^{2}} \int_{\infty}^{(0+)} \oint_{\{0, t\}} \frac{e^{a t} y(\tau, \lambda)}{\tau-t}\left(\frac{t}{\tau}\right)^{N-\frac{1}{2}} d \tau d t \\
& \sim \frac{\sqrt{2 \pi}}{(2 \pi i)^{2}} a^{\frac{1}{2}-N}\left[F^{(2)}\left(a ; \begin{array}{cc}
N+1, & 1 / 2 \\
2 \pi i, \lambda-1-\ln \lambda
\end{array}\right)+F^{(2)}\left(a ; \begin{array}{c}
N+1, \\
-2 \pi i, \lambda-1-\ln \lambda
\end{array}\right)\right] \\
& \quad-\frac{a^{\frac{1}{2}-N}}{2 \pi i} \sum_{n=0}^{\infty} c_{n}(\eta)\left[F^{(1)}\left(\begin{array}{c}
a-n \\
2 \pi i
\end{array}\right)-F^{(1)}\left(a ; \begin{array}{c}
N-n \\
-2 \pi i
\end{array}\right)\right]
\end{aligned}
$$


where

$$
\begin{aligned}
& F^{(1)}\left(\begin{array}{c}
a ; \\
\pm 2 \pi i
\end{array}\right)=\int_{0}^{ \pm \infty i} \frac{e^{ \pm 2 \pi i t} t^{N-n-1}}{a-t} d t \\
& =-(-a)^{N-n-1} \Gamma(N-n) e^{ \pm 2 \pi i a} \Gamma(n+1-N, \pm 2 \pi i a), \\
& F^{(2)}\left(a ; \begin{array}{l}
N+1, \\
\pm 2 \pi i, \lambda-1-\ln \lambda
\end{array}\right)=\int_{0}^{ \pm \infty i} \int_{0}^{\infty} \frac{e^{ \pm 2 \pi i t+(\lambda-1-\ln \lambda) \tau} t^{N} \tau^{-\frac{1}{2}}}{(a-t)(t-\tau)} d \tau d t .
\end{aligned}
$$

The computation of the hyperterminants $F^{(l+1)}(\cdots)$ is discussed in [5] and [7]. However, the occurrence of $\lambda-1-\ln \lambda$ in the $F^{(2)}$ hyperterminants in (4.2) makes the computation of these double integrals nontrivial. In fact, if we compare the $F^{(2)}$ hyperterminants in (4.2) with the left-hand side of (4.1), then we see that the $F^{(2)}$ hyperterminants depend on more (dominating) parameters and are more complicated. Hence, it is pointless to re-expand the remainder term $R_{N}(a, \lambda)$ in terms of hyperterminants. Other re-expansions in term of other functions might be possible, but it is very likely that one loses the simple resurgence.

Since some of the functions in the right-hand side of (4.2) are more complicated than the left-hand side of (4.1), we omit the proof of (4.2).

Acknowledgments. The author thanks the authors of [4] for the preprint of their paper, and is pleased to acknowledge the support of the Institute for Physical Science and Technology of the University of Maryland for a visit in 1997.

\section{References}

1. M. Abramowitz and I. A. Stegun, Handbook of Mathematical Functions with Formulas, Graphs, and Mathematical Tables, Nat. Bur. Standards Appl. Math. Series 55, U.S. Government Printing Office, Washington, D.C., 1964.

2. M. V. Berry and C. J. Howls, Hyperasymptotics for integrals with saddles, Proc. Roy. Soc. London, Ser. A 434 (1991), 657-675.

3. A. R. DiDonato and A. H. Morris, Jr., Algorithm 708. Significant digit computation of the incomplete beta function ratios, ACM Trans. Math. Softw. 18 (1992), 360-373.

4. T. M. Dunster, R. B. Paris, and S. Cang, On the high-order coefficients in the uniform asymptotic expansion for the incomplete gamma function, Methods Appl. Anal. 5 (1998), 223-247.

5. A. B. Olde Daalhuis, Hyperterminants I, J. Comp. Appl. Math. 76 (1996), 255-264.

6. Hyperasymptotic solutions of higher order differential equations with a singularity of rank one, Proc. Roy. Soc. London, Ser. A 454 (1998), 1-29.

7. _ Hyperterminants II, J. Comp. Appl. Math. 89 (1997), 87-95.

8. R. B. Paris and A. D. Wood, Stokes phenomenon demystified, Bull. Inst. Math. Appl. 31 (1995), 21-28.

9. N. M. Temme, The asymptotic expansion of the incomplete gamma functions, SIAM J. Math. Anal. 10 (1979), 757-766.

10. - Special Functions: An Introduction to the Classical Functions of Mathematical Physics, Wiley, New York, 1996.

11. Uniform asymptotics for the incomplete gamma functions starting from negative values of the parameters, Methods Appl. Anal. 3 (1996), 335-344.

12. R. Wong, Asymptotic Approximations of Integrals, Academic Press, New York, 1989.

Department of Mathematics and Statistics, King's Buildings, University of Edinburgh, EDINBURGH, EH9 3JZ, UK 